\title{
Prevalence of reactive attachment disorder in a deprived population
}

Helen Minnis, Susan Macmillan, Rachel Pritchett, David Young, Brenda Wallace, John Butcher, Fiona Sim, Katie Baynham, Claire Davidson and Christopher Gillberg

\section{Background}

Reactive attachment disorder (RAD) is associated with early childhood maltreatment and has unknown population prevalence beyond infancy.

\section{Aims \\ To estimate RAD prevalence in a deprived population of children.}

\section{Method}

All 1646 children aged 6-8 years old in a deprived sector of an urban UK centre were screened for RAD symptoms. Parents of high and low scorers were interviewed using semi-structured interviews probing for psychopathology and individuals likely to have RAD were offered face-to-face assessment.

\section{Results}

Questionnaire data were available from $92.8 \%$ of teachers and $65.8 \%$ of parents. Assessments were conducted with $50 \%$ of those invited and missing data were imputed - based on the baseline data - for the rest. We calculated that there would be 23 children with definite RAD diagnoses, suggesting that the prevalence of RAD in this population was $1.40 \%$ (95\% Cl 0.94-2.10).

\section{Conclusions}

In this deprived general population, RAD was not rare.

\section{Declaration of interest}

None.
Reactive attachment disorder (RAD) is a disorder of social functioning associated with abuse and neglect, with two subtypes: inhibited (wary, watchful behaviour) and disinhibited (overfriendly behaviour). It is persistent ${ }^{1}$ and is associated with significant psychiatric morbidity. ${ }^{2}$ This disorder has been described as 'extremely rare': ${ }^{3}$ Skovgaard has estimated prevalence to be $0.9 \%$ (95\% CI 0.1-3.4) in 1.5-year-olds, ${ }^{4}$ but prevalence is unknown beyond infancy. Despite preliminary attempts, large mental health surveys of school-age children have previously been unable to estimate the population prevalence of the disorder because appropriate measures were not available. ${ }^{3}$ Both screening and diagnostic measures for RAD in school-age children now exist ${ }^{5,6}$ and we report on the first population study of school-age children focusing on the prevalence of RAD.

\section{Method}

This was a three-phase study: phase 1 being a population questionnaire survey, phase 2 a nested case-control study using parental semi-structured interviews and phase 3 involving faceto-face assessments with children likely to have RAD (see Fig. 1). Our target population was all school children aged $6-8$ years old (i.e. school years primary 2 and 3 ) in a sector of a UK city chosen because of its high levels of deprivation. Because of the supposed rarity of $\mathrm{RAD}^{3}$ we involved a deprived population, in which child maltreatment may be more common, ${ }^{7}$ to increase the likelihood of finding individuals with the disorder. We were granted ethical approval for the study from the University of Glasgow and from education services in Glasgow City Council.

\section{Pilot study}

In two schools, focus groups were conducted with management staff and class teachers following completion of questionnaires, and qualitative information was gathered from parents during home visits. Information and questionnaire packs were translated into Arabic, Urdu and Somali. Based on the estimated prevalence in our pilot study (which we estimated as $1 \%$ ), we calculated that recruiting 1000 participants for phase 1 in the main study would give us a 95\% CI of 0.98-1.98 around our prevalence estimate. We predicted a $30-50 \%$ attrition rate for phase 1 .

\section{Main study}

All remaining schools in one large educational sector of the city were approached, via the Director of Education, with regard to participation and a presentation about the study was given to head teachers. All schools agreed to take part. As no changes in measures followed from the pilot study, we were able to include the pilot schools and our total mainstream school target sample therefore consisted of all 29 schools with a total of 1654 eligible children. Children whose birthdays fell within the 6-8 years age group attending specialist schools (for children with disabilities or emotional and behavioural disorders $(n=8)$ or living in out-of-area foster care $(n=27)$ were also approached (Fig. 1).

\section{Measures}

The Strengths and Difficulties Questionnaire (SDQ) is a 25-item parent- and teacher-report screening instrument investigating common child mental health problems. ${ }^{8}$ The SDQ has been well validated against other screening instruments ${ }^{8}$ and against psychiatric diagnosis. ${ }^{9}$ It contains subscales for emotional problems, conduct problems, hyperactivity, problems with peer relationships and prosocial behaviour.

The Relationship Problems Questionnaire (RPQ) is a 10-item parent- and teacher-report screening instrument for RAD symptoms. ${ }^{5}$ In a large general population twin sample, the RPQ had good internal consistency (Cronbach's apha $(\alpha)=0.85$ ) and factor analysis identified that six items describe inhibited RAD behaviours and four items describe disinhibited RAD behaviours. ${ }^{5}$ Children with parent RPQ scores of seven or over were defined as 'likely cases' and, hence, were screened as positive. This cut-off point was chosen based on the distribution of RPQ scores in a previous study of over 13000 7- to 8-year-old children from a general population twin study ${ }^{5}$ and (unpublished) cluster analysis of these data.

The DAWBA (Development and Wellbeing Assessment) ${ }^{10}$ is an online computerised parent-report interview, which investigates the common emotional, behavioural and hyperactivity disorders, 


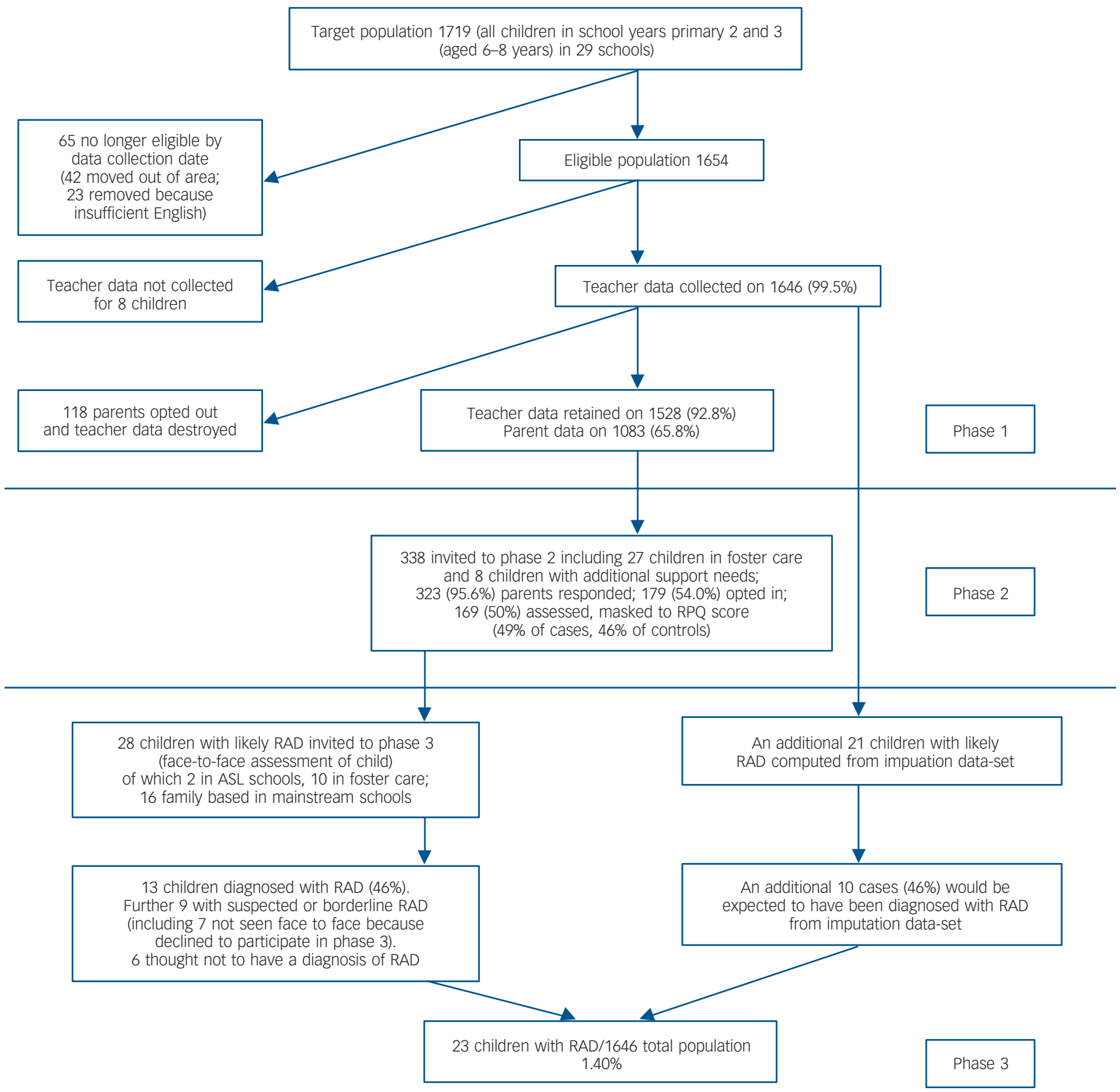

\section{Fig. 1 Study flow chart.}

$\mathrm{RPQ}$, Relationship Problems Questionnaire; RAD, reactive attachment disorder, ASL, additional support for learning.

and generates DSM-IV ${ }^{11}$ and ICD-10 ${ }^{12}$ diagnoses. It has been validated against clinician diagnosis and used in large-scale population research. ${ }^{10}$

The Child and Adolescent Psychiatric Assessment, Reactive Attachment Disorder module (CAPA-RAD) is a parental semistructured interview investigating symptoms of RAD. ${ }^{6}$ It contains 28-items for parents and its format is based on the CAPA - a well-validated, semi-structured parent-report interview for child psychopathology used in large epidemiological studies. ${ }^{13}$

The Observational Checklist for Reactive Attachment Disorder ${ }^{14}$ was originally developed for use in a clinic waiting room and explores interactions between the child and stranger(s) on first meeting. It has good internal consistency (Cronbach $\alpha=0.75$ ). In a previous study, it had good specificity but modest sensitivity in detecting children with $\mathrm{RAD}^{6}{ }^{6}$ therefore we have since used it as an adjunct to our parent-report diagnostic measure when making a diagnosis. It was modified, during the pilot study, for use at home, and was completed if the child was at home during the phase 2 assessment and/or at the school assessment.

The Scottish Index of Multiple Deprivation (SIMD) ranks small postcode areas in quintiles according to deprivation from one (most deprived) to five (least deprived). The SIMD is derived from 38 indicators across 7 domains: income, employment, health, education, skills and training, housing, geographic access and crime (www.scotland.gov.uk/Topics/Statistics/SIMD/ BackgroundMethodology).

\section{Phase 1}

Schools distributed a questionnaire pack to every child in school years primary 2 and 3 (age range 6-8 years). We did not to offer 
translated questionnaires because so few families returned nonEnglish questionnaires in the pilot $(n=1)$. The pack consisted of a cover letter from the school, a study information sheet with a form allowing parents to opt out of the study, and the questionnaire booklet containing the SDQ and RPQ. Parents were asked to complete either the questionnaire or the opt-out form and return it to the class teacher in a sealed envelope to be collected by the researcher. Parents who returned a questionnaire were entered into a prize draw to win shopping vouchers.

Researchers contacted non-responders by telephone and offered to post another questionnaire pack to participants, complete the questionnaire over the telephone, or accept a verbal opt out. Each non-responding family was telephoned at least three times, once in the morning, afternoon and evening.

Simultaneously, on a prespecified date, class teachers completed the questionnaires for each child in their class after brief training. Teacher questionnaires relating to children whose parents subsequently returned written opt-out forms were shredded and not included in the analysis $(n=118)$. Teacher questionnaires were retained for those children whose parents opted in, and those who remained non-responders $(n=1528$; $92.8 \%$, Fig. 1). If a school indicated that a family spoke no English, and they did not return a questionnaire, they were removed from the phase 1 sample pool $(n=23)$.

To establish the representativeness of our questionnaire sample, SIMD scores were obtained for both responders and non-responders. We also compared our study data with population norms for the SDQ (aggregate data in Ford et al: ${ }^{15}$ data disaggregated into relevant age bands kindly provided by Dr Tamsin Ford).

\section{Phase 2}

The sampling frame for phase 2 consisted of all children who screened positive (a score of seven or higher) on the RPQ according to either teachers or parents, and a random 1:1 sample of children who scored within normal ranges on the RPQ. We automatically included all children living in foster care $(n=27)$ in phase 2 because the prevalence of RAD is thought to be considerably higher in this population. ${ }^{3}$ Once the sampling frame was determined $(n=338)$, identification numbers were put on a second database and organised into school groups, masking researchers as to whether the child was in the high-scoring or randomly selected low-scoring group.

All potential participants for phase 2 were contacted initially by telephone, then the phase 2 pack, containing an information sheet, opt-in and opt-out form with a prepaid return envelope, was posted. Non-responders were called at least three times spanning morning, afternoon and early evening: 118 parents (35\%) required follow-up telephone calls. The researchers then visited the homes of those remaining non-responders, again at least three times per family spanning morning, afternoon and evening. A total of 44 parents (13\%) required recruitment visits. If the family was not in, a short letter was left, stating that a researcher had visited, would do so again to find out whether they were interested in taking part and encouraging them to contact us on a number provided to leave a confidential message with the secretary if they did not wish to be contacted again. Eleven families $(3 \%)$ were uncontactable after six visits, despite confirming address details with the school. If the parent had agreed to take part, the research team were more persistent - in one case visiting the parent 12 times before desisting. Interviews were conducted at home by non-clinical graduate psychologists. Interpreters were used, if required, to complete phase 2 assessments $(n=4)$. Interviews were clinically rated by senior child psychiatry trainees under the supervision of an experienced consultant child and adolescent psychiatrist, H.M. Any diagnostic dilemmas were discussed in group conferences led by H.M. $(n=42,25 \%)$.

\section{Phase 3}

Children were invited to phase 3 if their parents had reported key DSM-IV RAD symptoms on the CAPA-RAD interview performed in phase 2. We did not conduct phase 3 assessments on children who were not thought likely to have RAD because in previous research ${ }^{6}$ our assessment package identified no false positives in typically developing children.

For disinhibited RAD the key CAPA-RAD questions were:

(a) seeking comfort from strangers;

(b) indiscriminate friendliness;

(c) demanding and attention-seeking behaviour;

(d) minimal checking in unfamiliar settings;

(e) cuddliness with strangers, asking personal questions of strangers;

(f) invading social boundaries.

For inhibited RAD these were:

(a) avoids eye contact;

(b) frozen watchfulness;

(c) hypervigilance;

(d) unpredictable reunion responses.

Of the 28 children identified for phase 3, 22 (79\%) parents gave consent for their child to be assessed. These 22 children were seen by a researcher in school or, if in foster care out-of-area, in the foster home. Observations of child behaviour were made using the Observational Checklist for Reactive Attachment Disorder. Assessments were conducted in a private room and were videoed for diagnostic purposes.

\section{RAD diagnoses}

Diagnoses of RAD were made, based on DSM-IV criteria, by H.M. and the research team, following review of the CAPA-RAD, the teacher RPQ, the Observational Checklist for Reactive Attachment Disorder, comorbid diagnoses (from the DAWBA) and videotaped interaction between the child and researcher (who was a stranger to the child at the assessment visit). We have used this method of diagnosing RAD in previous research and have shown that it is highly sensitive and specific in discriminating children with RAD from typically developing children. ${ }^{6}$ Where the diagnosis was not absolutely clear, or where we were unable to see the child in school and were relying simply on interview and questionnaire data, the child was given a 'borderline/suspected' diagnosis. Both DSM-IV and ICD-10 suggest that RAD should only be diagnosed in the presence of a history of 'pathogenic care'. We decided that it would be upsetting for participants and reduce response rates if we asked parents direct questions about abuse and neglect of their child, although this was explored in the post-traumatic stress disorder (PTSD) section of the DAWBA, which included abuse as an example of a trauma. Examples of maltreatment reported in this section included physical abuse and witnessing domestic violence.

\section{Statistical methods}

Although we had no bias in the selection of schools (because all agreed to take part) and almost full screening data from teachers on our population (teacher SDQ and RPQs), only $50 \%$ of our invited sample took part in the second (case-control) phase. It 
is likely that there was some bias in our sampling at this stage, therefore we wished to estimate the number of cases that would have been ascertained if we had also managed to assess the other $50 \%$ of our population. The first step was to establish the sensitivity and specificity of our algorithm for deciding that a child had a likely diagnosis of RAD. We performed binary logistic regression, using the total teacher RPQ score, the total score for core CAPA-RAD disinhibited items and the total score for core CAPA-RAD inhibited items. A receiver operating characteristic (ROC) analysis was performed to determine the optimum sensitivity and specificity when determining RAD caseness. In order to apply the logistic regression model to the entire dataset and identify likely cases of RAD, we imputed CAPA-RAD scores for the missing $50 \%$ of our phase 2 sample, based on the variables for which we had virtually full data.

For the multiple imputation, the following variables were identified as having virtually full data: whether or not the child was selected as a case or control for phase 2, gender, SIMD and teacher RPQ. These were used to impute missing values for the core CAPA-RAD inhibited and disinhibited items listed earlier. In total, 100 data-sets were imputed with constraints added to ensure the values predicted in the multiple regression on the predictive variables were within the correct limits. Once the total inhibited and disinhibited CAPA-RAD parameters had been estimated from the individual components, the predictive logistic regression model was used to classify each of the participants as probable cases/controls.

\section{Results}

We obtained completed questionnaires from teachers on 1646 children (99.5\%). After parental opt-outs were taken into consideration, we were able to retain data from teachers on 1528 children (92.8\%). We received responses from 1227 parents $(74.5 \%)$ including 118 written opt-outs $(7.2 \%), 26$ declined to complete the questionnaire by telephone and 1083 completed questionnaires $(65.8 \%)$.

We managed to assess $50 \%$ of those families invited to phase 2 . For many families of both case and control children, achieving the phase 2 data collection required multiple telephone calls and/or visits in order to reach those who had given opt-in consent (see Method). All those we contacted were happy with our repeated attempts at contact when eventually a successful appointment was achieved.

\section{Representativeness of the sample}

Although SDQ scores were higher than the general UK population in our sample (mean (s.d.) parent total difficulties in the UK $v$. study population $(8.69$ (5.94) v. $9.30(5.95)$ and teacher total difficulties UK $v$. study population 6.78 (5.70) v. 7.67 (6.15)), the only statistically significant difference related to teacher scores (parent $t=1.59$ (d.f. $=1282$ ), $P=0.12$; teacher $t=2.18$ (d.f. $=1740), P<0.05)$.

The mean teacher SDQ total difficulties score was slightly higher in those children whose parents did not return a parent SDQ, but did not opt out compared with that in the group whose parents did return a parent SDQ $(8.04 v .7 .49)$, but this difference was not statistically significant $(t=1.6($ d.f. $=1479), P=0.11)$. As children attending local primary schools usually come from postcode areas near the school, we used 'school SIMD' as a proxy measure for those families for whom we did not have other data such as street address. The median school quintile was equal to 1 for both children whose parents did and did not return a questionnaire in phase 1 , including those who chose not to take part in the study by returning an opt-out form $(n=118)$.

\section{Estimate of prevalence}

The phase 2 parental interviews with the DAWBA and CAPA-RAD identified 28 children likely to have RAD from the 50\% of the sample who took part in phase 2. The ROC analysis identified a probability of caseness of 0.57 , derived from the logistic regression, as the optimal cut-off point. Using this cut-off correctly identified 25 out of 28 likely cases (sensitivity $89.3 \%$ ) and correctly identified 128 out of 129 as non-cases (specificity 99.2\%) (one child in the phase 2 control group was eventually diagnosed with RAD and had an RPQ score of six when our cut-off was seven). In order to estimate the prevalence in the total population, multiple imputation was performed. The first five imputed data-sets are shown in online Table DS1. The predictive logistic regression model was then used to classify each of the case/ control participants as probable cases or non-cases. The results are shown in online Table DS2.

Of the 28 children in our original phase 3 data-set, we identified 13 children (46\%) as having a definite diagnosis of RAD after a full face-to-face assessment (including review of teacher RPQ, CAPA-RAD, comorbid DAWBA diagnoses, video material and completion of the structured observational checklist) and $6(21 \%)$ as not having RAD. We identified a further nine children $(32 \%)$ as having borderline or suspected RAD. Seven of these children were not assessed face to face, but all had apparently clear symptoms of RAD on the CAPA-RAD and teacher RPQ. The other two still had equivocal diagnostic status despite full data.

Extrapolating this to our imputed data-set, we would therefore expect $46 \%$ of our likely cases to have definite RAD and $79 \%$ to have definite or suspected/borderline RAD. From a total of 49 likely RAD cases, we would expect to have 23 definite cases ( $47 \%$ of the 49 ) and 39 cases if the suspected/borderline cases are included ( $80 \%$ of the 49). From our total population of 1646 , we estimate the prevalence of $\mathrm{RAD}$ to be $1.40 \%$ (95\% CI $0.94-2.10)$ and, when suspected/ borderline cases are included, to be $2.37 \%$ (95\% CI 1.74-3.22).

\section{Characteristics of children with definite or borderline/suspected RAD}

Of the 13 children with a definite diagnosis of RAD, 1 child had a diagnosis of inhibited RAD, whereas the others had disinhibited RAD. All had at least one other likely comorbid diagnosis as defined by the DAWBA (some children had more than one comorbid diagnosis): 7 (54\%) had a likely diagnosis of attention-deficit hyperactivity disorder (ADHD); 3 (23\%) oppositional defiant disorder; $4(31 \%)$ conduct disorder; 2 (15\%) PTSD; 2 (15\%) autism spectrum disorder (ASD); 4 $(31 \%)$ a specific phobia and $1(8 \%)$ a tic disorder. This was compared with a low prevalence of disorder in the low-scoring comparison group in which no children had a diagnosis of oppositional defiant disorder, conduct disorder, PTSD, ASD, or tic disorder, 1 child $(2.4 \%)$ had ADHD (Fisher's exact test $P=0.058)$ and 2 children (5\%) had specific phobia (Fisher's exact test $P=0.089)$. Of the 22 children with definite or suspected/borderline RAD assessed, 10 (45.5\%) were looked after and accommodated children living in foster care. The other 12 $(54.5 \%)$ were living with their birth families. All but one of the children with a definite diagnosis of RAD had histories of definite or suspected maltreatment documented during interviews with parents or carers and all but two of those with a borderline/ suspected diagnosis of RAD had such a history. In the other three, a history of maltreatment was impossible to determine but may well have been present: we made a child protection referral regarding one child for whom there was no clear history of maltreatment. For none of these three children were their RAD symptoms explicable by any other disorder. 


\section{Discussion}

The prevalence of RAD was found to be $1.40 \%$ in this deprived urban population, which is considerably higher than previously believed. It was extremely challenging to actually meet families at home: for some families numerous telephone calls and visits were required to achieve a visit that parents had invited us to make. This has important implications for service provision for children with RAD and children with other psychiatric disorders living in deprived areas, as no currently configured child and adolescent mental health service would have the resources to make such assertive outreach to families.

Although the great majority of children diagnosed with RAD had a history of maltreatment, we were unable to confidently establish that all had been maltreated, as it would have been too intrusive to enquire further than we did about this within a school-based population study. This underlines the problems inherent in a history of maltreatment being part of the diagnosis of RAD, yet this requirement is likely to be retained in DSM-V (www.dsm5.org/).

Children diagnosed with RAD had a range of comorbid diagnoses, some of which had been previously unrecognised by services. Children with RAD are likely to continue to have a range of potentially disabling difficulties throughout childhood, even if placed in nurturing adoptive families, ${ }^{16}$ therefore it is essential that they are identified and offered services.

\section{Limitations}

Our study is limited by our $65.7 \%$ parental response rate, however the social demographics of responders and non-responders were very similar so our sample appears to be reasonably representative of our population. Although we only managed to conduct parental interviews with $50 \%$ of those we invited, we were able to impute data from teacher questionnaires and demographic data on almost the entire population and we are therefore reasonably confident in our estimate of prevalence in this deprived population. The sensitivity and specificity of our algorithm for likely RAD was good, but it did miss one case of RAD - a child with an RPQ score of six when our cut-off was seven. It may be that a cut-off point for likely diagnosis of six would be more appropriate for future research. Our observational measurement of RAD behaviours was made in school for the majority of the sample but at home for children in foster care and it will be important to explore these measurement issues in future research. It is unknown whether this population prevalence would be applicable elsewhere, therefore further prevalence studies are needed in rural and urban, deprived and more affluent populations.

\section{Clinical implications}

It appears that, at least in a deprived population, RAD is not a rare condition in school-age children. However, assertive outreach was required in order to conduct interviews with the families, suggesting that children with RAD - along with children with other psychiatric disorders in deprived populations - may be a hidden group. Children with RAD had complex difficulties with a range of comorbid diagnoses and it will be important for child and adolescent mental health services to develop assertive strategies to reach this vulnerable group of children.

\section{Funding}

The study was funded by the Chief Scientist Office of the Scottish Government and the Gillberg Neuropsychiatry Centre.

\section{Acknowledgements}

Thanks are due to all participating families and schools who so enthusiastically supported the study. We also thank Drs Jane Fuller, Laxmi Kathuria, Michelle Quilter, Tania Schumm, Tina Irani, Muhammad Ather and Manju Haridas for conducting clinical ratings and Dr Tamsin Ford for providing us with data for comparison.

Helen Minnis, FRCPsych, PhD, Susan Macmillan, MA, Rachel Pritchett, MA Institute of Mental Health \& Wellbeing, University of Glasgow; David Young, PhD, Department of Mathematics and Statistics, University of Strathclyde, Glasgow; Brenda Wallace, John Butcher, Education Services, Glasgow City Council; Fiona Sim, BSC, Katie Baynham, BSc, Claire Davidson, BA, Christopher Gillberg, PhD $\mathrm{MD}$, Institute of Mental Health \& Wellbeing, University of Glasgow, UK

Correspondence: Helen Minnis, Institute of Mental Health \& Wellbeing, University of Glasgow, Caledonia House, Royal Hospital for Sick Children, Yorkhill, Glasgow G3 8SJ, UK. Email: Helen.Minnis@glasgow.ac.uk

First received 27 Apr 2012, final revision 21 Dec 2012, accepted 21 Jan 2013

\section{References}

1 Gleason MM, Fox NA, Drury S, Smyke A, Egger HL, Nelson CS, et al. Validity of evidence-derived criteria for reactive attachment disorder: indiscriminately social/disinhibited and emotionally withdrawn/inhibited types. J Am Acad Child Adolesc Psychiatry 2011; 50: 216-31.

2 Rutter M, Colvert E, Kreppner J, Beckett C, Castle J, Groothues C, et al. Early adolescent outcomes for institutionally-deprived and non-deprived adoptees. I: disinhibited attachment. J Child Psychol Psychiatry 2007; 48: 17-30.

3 Meltzer $\mathrm{H}_{1}$ Gatward R, Corbin T, Goodman R, Ford T. The Mental Health of Young People Looked After by Local Authorities in England. The Report of a Survey carried out in 2002 by Social Survey Division of the Office for National Statistics on behalf of the Department of Health. TSO (The Stationery Office), 2005

4 Skovgaard AM. Mental health problems and psychopathology in infancy and early childhood. An epidemiological study. Dan Med Bull 2010; 57: B4193.

5 Minnis H, Reekie J, Young D, O'Connor T, Ronald A, Gray A, et al. Genetic, environmental and gender influences on attachment disorder behaviours. Br J Psychiatry 2007; 190: 495

6 Minnis H, Green J, O'Connor T, Liew A, Glaser D, Taylor E, et al. An exploratory study of the association between reactive attachment disorder and attachment narratives in early school-age children. J Child Psychol Psychiatry 2009; 50: 931-42.

7 Radford L, Corral S, Bradley C, Fisher H, Bassett C, Howat N, et al. Child abuse and Neglect in the UK Today. NSPCC, 2011.

8 Goodman R, Scott S. Comparing the Strengths and Difficulties Questionnaire and the Child Behavior Checklist: is small beautiful? J Abnorm Child Psychol 1999; 27: 17-24.

9 Goodman R, Ford T, Simmons H, Gatward R, Meltzer H. Using the Strengths and Difficulties Questionnaire (SDQ) to screen for child psychiatric disorders in a community sample. Int Rev Psychiatry 2003; 15: 166-72.

10 Goodman R, Ford T, Richards H, Gatward R, Meltzer H. The development and well-being assessment: description and initial validation of an integrated assessment of child and adolescent psychopathology. J Child Psychol Psychiatry 2000; 41: 645-56.

11 American Psychiatric Association. Diagnostic and Statistical Manual of Mental Disorders (4th edn) (DSM-IV). APA, 1994.

12 World Health Organization. The ICD-10 Classification of Mental and Behavioural Disorders: Clinical Descriptions and Diagnostic Guidelines. WHO, 1992.

13 Angold A, Costello EJ. The Child and Adolescent Psychiatric Assessment (CAPA). J Am Acad Child Adolesc Psychiatry 2000; 39: 39-48.

14 McLaughlin A, Espie C, Minnis H. Development of a brief waiting room observation for behaviours typical of reactive attachment disorder. Child Adolesc Men Health 2010; 15: 73-9.

15 Ford T, Goodman R, Meltzer H. Service use over 18 months among a nationally representative sample of British children with psychiatric disorder. Clin Child Psychol Psychiatry 2003; 8: 37-51.

16 Kocovska E, Puckering C, Follan M, Smillie M, Gorski C, Barnes J, et al. Neurodevelopmental problems in maltreated children referred with indiscriminate friendliness. Res Dev Disabil 2012; 33: 1560-5. 